\title{
Apparent Non-Newtonian Behavior of Ionic Liquids
}

\author{
Agnès Piednoir, ${ }^{\dagger}$ Audrey Steinberger ${ }^{\ddagger}{ }^{\circ}$ Cécile Cottin-Bizonne, ${ }^{\dagger}$ and Catherine \\ Barentin $^{*, \dagger}$ \\ $\dagger$ †niversité de Lyon, Université Claude Bernard Lyon 1, CNRS, Institut Lumière Matière, \\ F-69622, VILLEURBANNE, France \\ $\ddagger$ Univ Lyon, ENS de Lyon, Univ Claude Bernard, CNRS, Laboratoire de Physique, Lyon, \\ France
}

E-mail: catherine.barentin@univ-lyon1.fr 


\begin{abstract}
A significant viscosity variation with the shear rate has been observed for several ionic liquids in rheometry experiments above a critical shear rate. Depending on the liquid and the rheological conditions, both viscosity increase and decrease have been reported. So far, these variations have been interpreted as a signature of a non-Newtonian behavior. However, the measured critical shear rates are orders of magnitude below the ones predicted by numerical simulations. In this work, we perform new rheometry experiments with both ionic liquids and Newtonian liquids to elucidate this discrepancy. For these two types of liquids, both a viscosity decrease and an increase have been measured depending on the geometry of the rheometer and the zero-shear viscosity of the liquid. We interpret the viscosity decrease as resulting from viscous heating, since the viscosity of the investigated liquids is also highly temperature-dependent, and the viscosity increase as resulting from the development of instabilities at high shear rates.
\end{abstract}

Ionic liquids (ILs) have attracted great interest in the past few years. They have been seen as opening new frontiers in material science ${ }^{1}$ from promising alternatives to traditional industrial solvents ${ }^{2}$ and new lubricants. ${ }^{3,4}$ This has inspired a large number of experimental studies, simulations and theoretical studies in the recent years. ${ }^{5-7}$ Here, we focus on the mechanical properties of ILs. Their viscosities are classically measured by the falling sphere method, the Stabinger device, ${ }^{8}$ or flows in capillary tubes. ${ }^{9}$ These methods allow the exploration only a small range of solicitations, whereas viscosity may depend on the applied stress or shear rate. ${ }^{10-12}$ A liquid whose viscosity is constant with the applied stress or shear rate is considered as Newtonian. Otherwise, the liquid is considered as a non-Newtonian fluid. A decrease (resp. increase) of the viscosity with the shear rate is called shear-thinning (resp. shear-thickening). Shear-thinning observed above a critical shear rate $\dot{\gamma}_{c}$ for complex fluids is generally understood as a consequence of an alignment of aggregates ${ }^{13,14}$ inducing a decrease in dissipation. Shear-thickening is often attributed to the solid friction between particles ${ }^{15-18}$ inducing an increase in dissipation. Therefore the analysis of such non-Newtonian effects allows the exploration of some properties of the system such as the interaction between 
particles or the microstructure. Indeed, the knowledge of the relaxation time ${ }^{14}$ associated with the critical shear rate $\dot{\gamma}_{c}$ gives access to the characteristic size of the microstructure. Both shear-thinning and shear-thickening effects have been reported experimentally ${ }^{19-22}$ for several ILs at shear rates above typically $10^{2}-10^{4} \mathrm{~s}^{-1}$. In Burrel et al.'s work, ${ }^{19}$ a transition between both behaviors has even been shown for the same ionic liquid by varying the temperature. Shear-thinning has also been reported numerically ${ }^{10,12}$ for shear rates above typically $10^{7}-10^{9} \mathrm{~s}^{-1}$. However, 5 orders of magnitude separate the experimental and numerical results for the shear-thinning effect, and there is no theoretical explanation of the observed shear-thickening behavior. Understanding this huge difference in critical shear rate for the shear-thinning, as well as the transition between shear-thinning and shear-thickening behaviors motivates further rheological experiments on IL.

In this work, we study the properties of 1-alkyl-3-methylimidazolium bis(trifluoromethylsulfonyl)imide, denoted $\left[\mathrm{C}_{n} \operatorname{mim}\right][\mathrm{NTF} 2]$ with $n=1,2,4,8,10$ indicating the length of the alkyl chain, purchased from Solvionic. The viscosity of $\left[\mathrm{C}_{n} \mathrm{mim}\right][\mathrm{NTF} 2]$ is measured with a controlled-stress rheometer (AntonPaar MCR301). The dependence of the viscosity on the shear rate, ranging from 5 to $5000 \mathrm{~s}^{-1}$, is explored using different geometries (couette, cone-plate and plate-plate). The dependence of the viscosity on the temperature ranging from 273 to $333 \mathrm{~K}$ is also explored in the Couette geometry, the temperature being imposed by a Peltier device at the stator. The viscosity of a Newtonian fluid (water/glycerol mixture) in the same rheological conditions (shear rates, temperatures, geometry) is also measured as a benchmark to avoid any misinterpretation of the experimental data. ${ }^{23,24}$ In the main text, we present only the results obtained with $\left[\mathrm{C}_{10} \mathrm{mim}\right][\mathrm{NTF} 2]$ whose molecular structure has been investigated theoretically. ${ }^{25,26}$ Results obtained with other ILs $\left[\mathrm{C}_{n}\right.$ mim] $[\mathrm{NTF} 2]$ are collected in the Supporting Information and are similar to those of $\left[\mathrm{C}_{10} \mathrm{mim}\right][\mathrm{NTF} 2]$. The first part of the paper is devoted to the study of the shear-thinning effect and the second part to the shear-thickening effect.

In the first part, we measure the viscosity of $\left[\mathrm{C}_{10} \mathrm{mim}\right][\mathrm{NTF} 2]$ with a Couette geometry 
(100 $\mu \mathrm{m}$ gap) as a function of the shear rate $\dot{\gamma}$ and for several temperatures $T$. For any studied temperature from $273 \mathrm{~K}$ to $333 \mathrm{~K}$, the viscosity displays a plateau at low shear rates, whose value is called zero-shear viscosity $\eta_{0}$. The viscosity then decreases at higher shear rate, above a critical shear rate denoted $\dot{\gamma}_{c}$. These viscosity decreases are well fitted by a Carreau law: $\eta(\dot{\gamma}) / \eta_{0}=\left(1+\left(\dot{\gamma} / \dot{\gamma}_{c}\right)^{2}\right)^{-\alpha}$, which allows to define the value of the critical shear rate $\dot{\gamma}_{c}$ for each curve (see the Supporting Information). The exponent $\alpha$ is of the order of 0.03 , typically 1 order of magnitude smaller than the ones found with classical shear-thinning systems. ${ }^{10,13}$ Concerning $\eta_{0}$, its value is calculated by averaging all data on the plateau region corresponding here to $\dot{\gamma} / \dot{\gamma}_{c}$ ranging from 0.04 to 0.3 . We represent in Figure 1a the normalized viscosities $\eta / \eta_{0}$ with respect to the normalized shear rate $\dot{\gamma} / \dot{\gamma}_{c}$. Note that both the zero-shear viscosity $\eta_{0}$ and $\dot{\gamma}_{c}$ depend on temperature, as shown in Figure 1b.
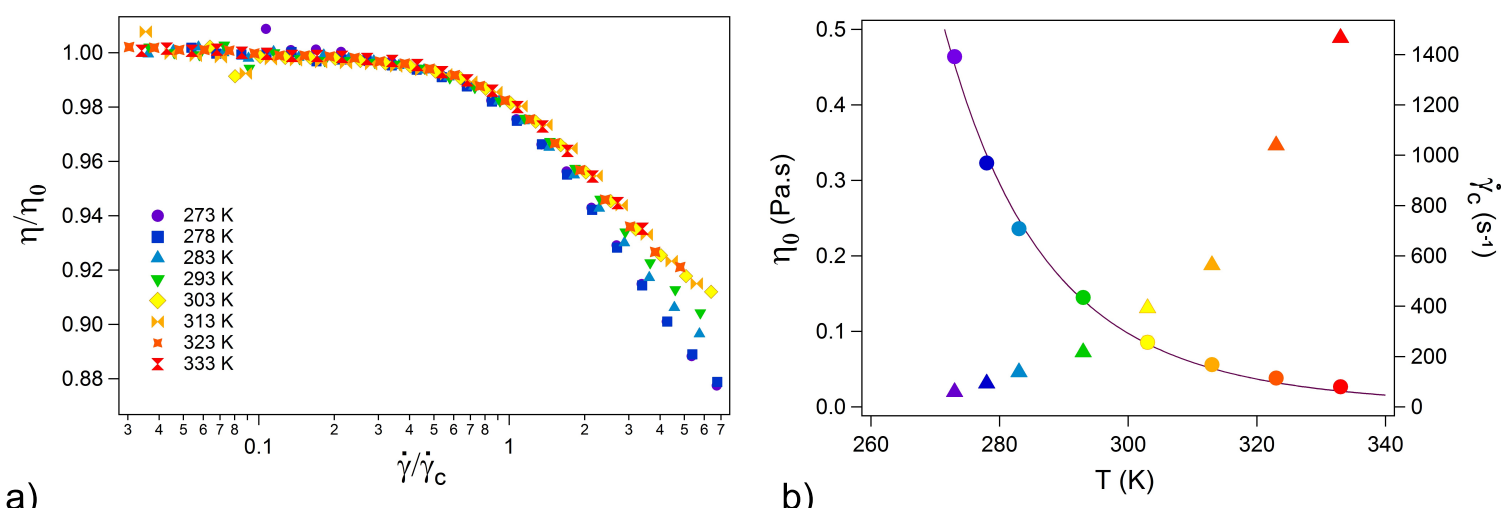

b)

Figure 1: a) Evolution of the normalized viscosity, $\eta / \eta_{0}$, with the normalized shear rate $\dot{\gamma} / \dot{\gamma}_{c}$ for the ionic liquid $\left[\mathrm{C}_{10} \mathrm{mim}\right][\mathrm{NTF} 2]$ for various temperatures from 273 to $333 \mathrm{~K}$, in a Couette geometry with a $100 \mu \mathrm{m}$ gap. b) For $\left[\mathrm{C}_{10} \mathrm{mim}\right][\mathrm{NTF} 2]$, critical shear rate $\dot{\gamma}_{c}$ (triangle) as a function of temperature $T$, and zero-shear viscosity $\eta_{0}$ (circle) including error bars (smaller than the symbol size) as a function of temperature $T$ and fit with $\eta_{0}(T)=$ $1.7 \times 10^{-8} \exp (4660 / T)$. The relative error for zero-shear viscosity ranges from 0.1 to $0.8 \%$ depending on the temperature.

A viscosity decrease with the shear rate has already been observed on various ILs by Kulkarni et al. ${ }^{20}$ for measurements carried out using a controlled-stress rheometer, with a critical shear rate typically above $\dot{\gamma}_{c}=200 \mathrm{~s}^{-1}$. Burrel et al. ${ }^{19}$ have also measured critical 
shear rates $\dot{\gamma}_{c}$ above $1000 \mathrm{~s}^{-1}$ on several ionic ILs using a controlled shear-rate rheometer. Both authors have interpreted it as a signature of a shear-thinning effect in these ILs, associated with the alignment of IL aggregates ${ }^{19}$ or to the disruption of a network of hydrophobic blocks. ${ }^{20}$ However, other experiments based on ultrasound attenuation ${ }^{27}$ and on shear impedance spectroscopy ${ }^{28}$ found relaxation times corresponding to critical shear rates above $10^{8} \mathrm{~s}^{-1}$ in several imidazolium-based IL. On the numerical side, shear-thinning has also been observed at similarly high critical shear rates (above $10^{7} \mathrm{~s}^{-1}$ ) in simulations of IL. ${ }^{10,12}$ These values of critical shear rates are however more than 4 orders of magnitude higher than the ones found in refs ${ }^{19,20}$ and in our rheological measurements.

From the knowledge of the critical shear rate, we can estimate a corresponding size $a$ for the aggregates. Indeed, shear tends to align the aggregates, whereas thermal fluctuations tend to make them rotate. Shear-thinning is then observed when $\dot{\gamma} \geq D_{\text {rot }}=\frac{k_{B} T}{8 \pi \eta_{0} a^{3}}$, where $D_{\text {rot }}$ is the Brownian rotational diffusion coefficient. We deduce that the typical aggregate size is $a=\left(\frac{k_{B} T}{8 \pi \eta_{0} \dot{\gamma}_{c}}\right)^{1 / 3}$. For a viscosity of $\eta_{0}=0.1$ Pa.s (typical of $\left[\mathrm{C}_{10} \mathrm{mim}\right][\mathrm{NTF} 2]$ at room temperature), a critical shear rate of $\dot{\gamma}_{c} \sim 200 \mathrm{~s}^{-1}$ suggests an aggregate size $a$ of around $20 \mathrm{~nm}$, corresponding to the aggregation of about a few tens of thousands of IL molecules, which is surprisingly huge. The same aggregate size around $20 \mathrm{~nm}$ is deduced from critical shear rates for all temperatures and all ILs we have probed (see Figure 1b and Supporting Information). Similar sizes of aggregates with other IL can be extracted in the same way from Kulkarni et $\mathrm{al}^{20}$ and Burrel et al. ${ }^{19}$ The fact that this size is independent of the nature of IL is also unexpected. On the numerical side ${ }^{12}$ much smaller aggregate sizes of around $a \simeq 0.8 \mathrm{~nm}$ are found, in agreement with the nano-heterogeneities measured experimentally with small-and-wide-angle X-ray scattering (SWAXS) in $\left[\mathrm{C}_{n} \mathrm{mim}\right][\mathrm{NTF} 2] .{ }^{29}$ These huge differences in aggregate sizes obtained on one side by rheological experiments and on the other side by simulations or SWAXS, ultrasound experiments and shear impedance spectroscopy raise doubts about the interpretation of the viscosity decrease observed in the rheological experiments in terms of shear-thinning. 
In the following, we suggest to interpret this viscosity decrease not as a result of a shear-thinning effect but as a result of heating effects instead. Viscous heating is possible whenever the viscosity $\eta_{0}$ is temperature-dependent, ${ }^{30}$ which is the case for IL (see refs ${ }^{8,19,20}$ and Figure 1b). The temperature rise $\Delta T$ due to shear has been estimated several decades ago $^{31}$ and scales as $\Delta T \simeq \frac{\eta_{0} \dot{\gamma}^{2} h^{2}}{\kappa}$ with $h$ the typical gap of the geometry and $\kappa$ the thermal conductivity. This temperature rise induces, at first order, a viscosity decrease $\Delta \eta$ given by: ${ }^{32}$

$$
\frac{\Delta \eta}{\eta_{0}}=\frac{1}{\eta_{0}} \frac{\partial \eta_{0}}{\partial T} \Delta T=\beta \frac{\Delta T}{T}
$$

where $\beta=\frac{T}{\eta_{0}}\left(\frac{\partial \eta_{0}}{\partial T}\right)_{T}$ is the thermal sensitivity. For $\left[\mathrm{C}_{10} \operatorname{mim}\right][\mathrm{NTF} 2]$, we measure $|\beta| \simeq 16$ at room temperature. From Equation 1, we expect the relative variation of viscosity to scale as:

$$
\left|\frac{\Delta \eta}{\eta_{0}}\right| \propto \dot{\gamma}^{2}
$$

With the aim to test this dependence, we plot in Figure 2 the absolute relative variation of the viscosity of $\left[\mathrm{C}_{10} \mathrm{mim}\right][\mathrm{NTF} 2]$ as a function of the normalized shear rate, for various temperatures. The experimental uncertainty on the relative variation of viscosity $(0.6 \%$ in average) sets the lower limit of the $\mathrm{Y}$-axis. For intermediate values of the relative variations of viscosity (typically smaller than 5-6\%), we observe a power law dependence with the shear rate whose slope is between 1 and 2 . It is slightly smaller than the slope of 2 predicted by Equation 2, which is not so surprising for two reasons. First, Equation 2 is only a first-order approximation that neglects the effect of the viscosity decrease on the magnitude of the viscous heating and is only valid for small relative viscosity variations. Second, the temperature increase is also limited by the thermal exchanges with the environment, including both the liquid bath and the Peltier element that controls the setpoint temperature. In particular, it is important to note that the volume of the sheared liquid in the $100 \mu \mathrm{m}$ gap Couette geometry corresponds to only $1 \%$ to the total liquid volume, which plays the role of 
a temperature reservoir. Finally, a saturation is observed at higher relative viscosity variations (around 10\%) which corresponds to a temperature increase of $\Delta T=\frac{T}{\beta} \frac{\Delta \eta}{\eta_{0}}=\frac{T^{2}}{4660}\left|\frac{\Delta \eta}{\eta_{0}}\right|$ comprised between $1.6 \mathrm{~K}$ (at $273 \mathrm{~K}$ ) and $2.4 \mathrm{~K}$ (at $333 \mathrm{~K}$ ). We think that it is due to the thermal regulation by the Peltier element. The fine modelisation of these thermal exchanges is however beyond the scope of this paper, since it is very dependant on the experimental geometry, the proportion of sheared liquid with respect to the non-sheared liquid, the details of the temperature control loop, and the position of the temperature sensor.

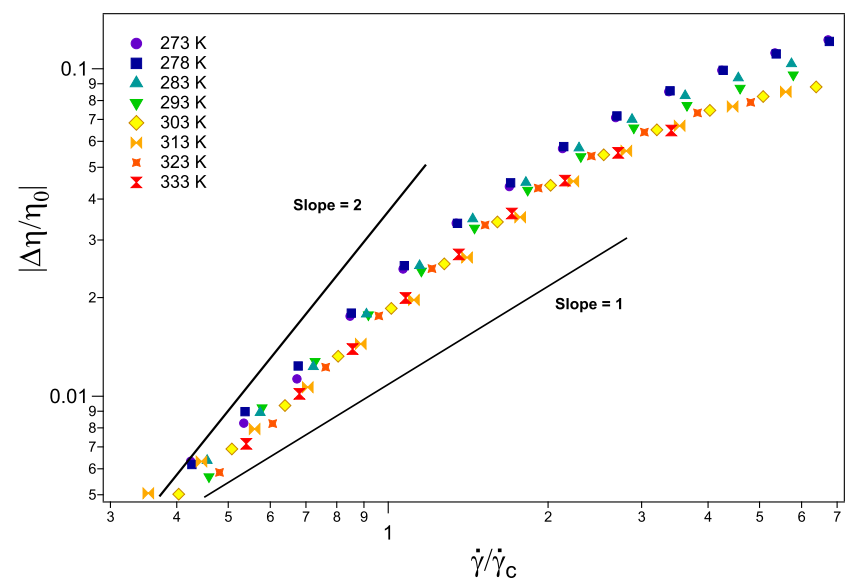

Figure 2: Evolution of the absolute relative variation of viscosity of $\left[\mathrm{C}_{10} \mathrm{mim}\right][\mathrm{NTF} 2]$ with the normalized shear rate $\dot{\gamma} / \dot{\gamma}_{c}$ at various temperatures ranging from $T=273$ to $333 \mathrm{~K}$.

To further check the validity of our viscous-heating assumption, we perform similar experiments with a Newtonian liquid, a water/glycerol (10/90) mixture. Its thermal sensitivity at room temperature $|\beta|=18$ and its zero-shear viscosity are similar to the ones of $\left[\mathrm{C}_{10} \mathrm{mim}\right][\mathrm{NTF} 2]$. We observe that the dependence of its viscosity on the shear rate (Figure 3a) is extremely similar to the one obtained with $\left[\mathrm{C}_{10} \mathrm{mim}\right][\mathrm{NTF} 2]$ (Figure 1a). In particular, the order of magnitude of the critical shear rate $\left(\sim 100 \mathrm{~s}^{-1}\right)$ is very similar. However, Newtonian liquids such as a water-glycerol mixture do not exhibit shear-thinning behavior at such a small range of shear rates at room temperature. ${ }^{23}$ Therefore, the only reasonable explanation for this viscosity decrease is the temperature increase induced by the mechanical shear.

From the knowledge of $\beta$ and from equation 1, we estimate the temperature increase in 

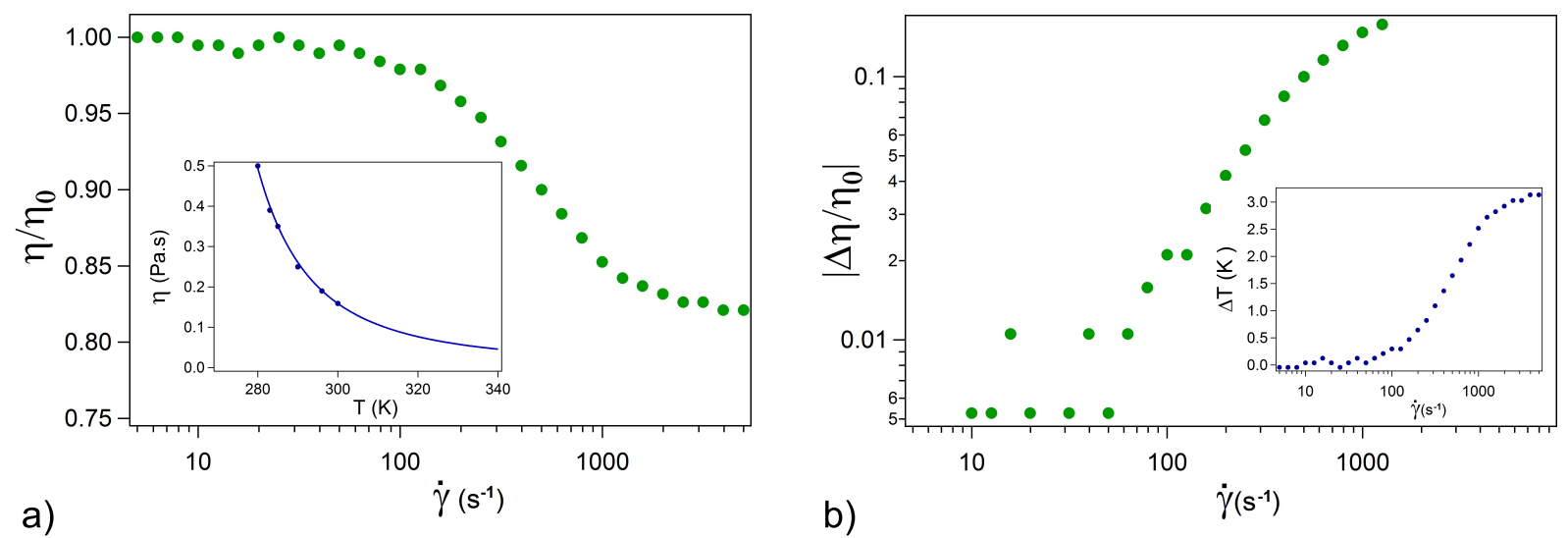

Figure 3: a) Evolution of the viscosity with shear rate for a water-glycerol (10/90) mixture at $\mathrm{T}=296 \mathrm{~K}$, in a Couette geometry with a $100 \mu \mathrm{m}$ gap; inset: evolution of $\eta_{0}$ with the temperature $T$ and fit with $\eta_{0}(T)=1.8 \times 10^{-9} \exp (5440 / T)$. b) Evolution of the absolute relative variation of viscosity of this water-glycerol mixture with the shear rate at $T=296 \mathrm{~K}$; inset: calculated temperature increase $\Delta T$ as a function of shear rate for the water-glycerol mixture.

the sheared liquid corresponding to the observed viscosity decrease for each shear rate. The inset of Figure 3b shows $\Delta T$ for the water-glycerol (10/90) mixture. A $10 \%$ variation of viscosity requires a temperature increase $\Delta T$ of the order of only $2 \mathrm{~K}$ within the sheared region. Similar temperature increases are estimated for $\left[\mathrm{C}_{10} \mathrm{mim}\right][\mathrm{NTF} 2]$, as shown in the Supporting Information.

Note that geometry matters for the viscosity decrease. A dependence of the critical shear rate on the geometry has been reported a long time ago, ${ }^{33,34}$ and more recently ${ }^{32}$ for Newtonian liquids. In all these studies, the viscosity drop was due to viscous heating. We have observed a similar dependence on the geometry for $\left[\mathrm{C}_{10} \mathrm{mim}\right][\mathrm{NTF} 2]$. In a Couette geometry with a $100 \mu \mathrm{m}$ gap, $\dot{\gamma}_{c} \sim 50 \mathrm{~s}^{-1}$ at $T=278 \mathrm{~K}$ (see Figure 1a); whereas for a $1 \mathrm{~mm}$ gap, $\dot{\gamma}_{c} \sim 500 \mathrm{~s}^{-1}$ at the same temperature (see Figure $4 \mathrm{a}$ ). Such a dependence on the geometric setup rules out the explanation that the critical shear rate is due to intrinsic bulk properties of IL and thus confirms the viscous-heating assumption on a thin layer around the rotor.

Note that, interestingly, performing experiments on $\left[\mathrm{C}_{10} \mathrm{mim}\right][\mathrm{NTF} 2]$ using a Couette geometry with a $1 \mathrm{~mm}$ gap and spanning temperatures, we could observe various behaviors 

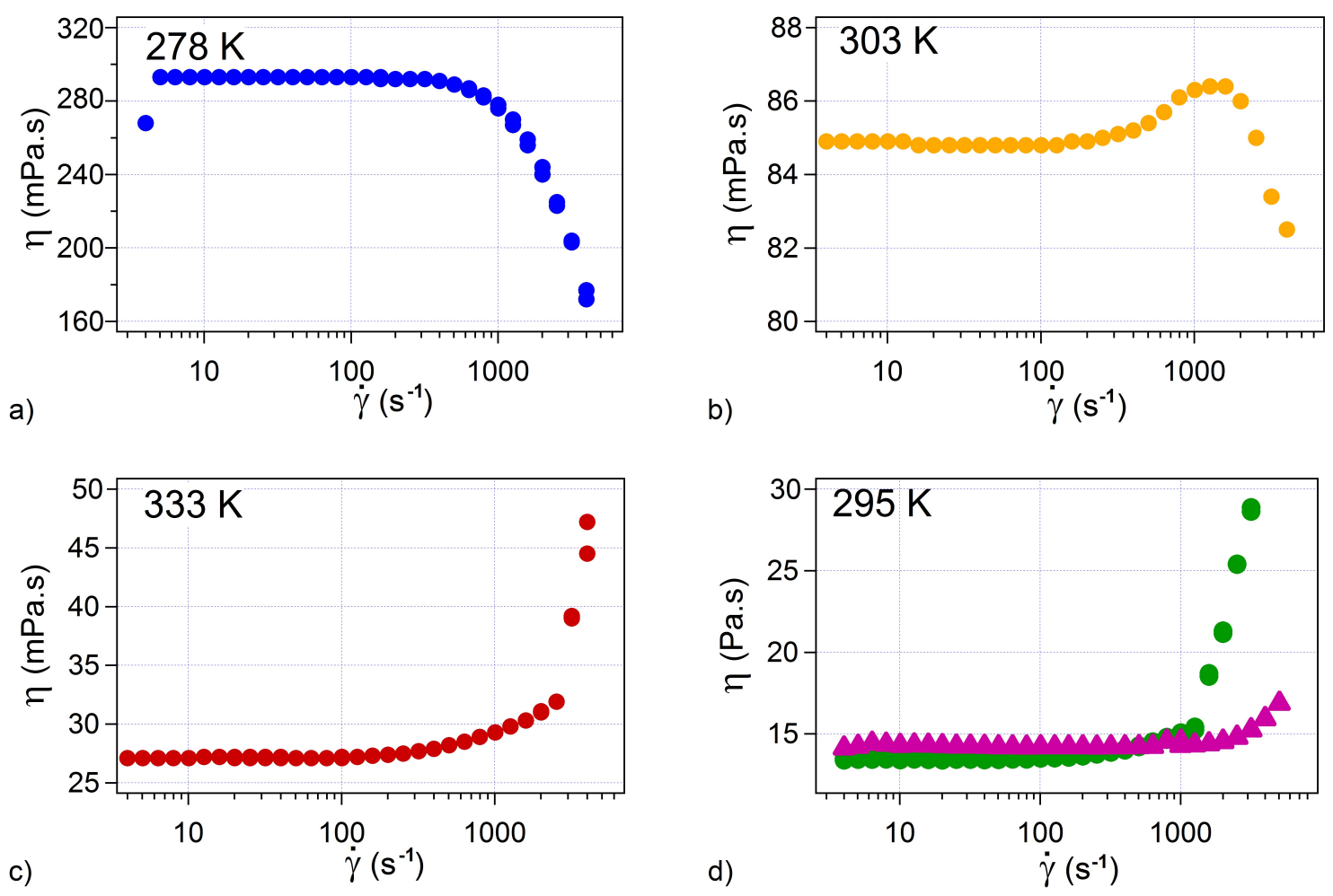

Figure 4: Evolution of the viscosity with shear rate for $\left[\mathrm{C}_{10} \mathrm{mim}\right][\mathrm{NTF} 2]$, in a Couette geometry with a $1 \mathrm{~mm}$ gap, a) at $\mathrm{T}=278 \mathrm{~K}$, b) at $\mathrm{T}=303 \mathrm{~K}$; c) at $\mathrm{T}=333 \mathrm{~K}$. d) Evolution of the viscosity with shear rate for a water-glycerol $(35 / 65)$ mixture, with $\eta_{0}=14 \mathrm{mPa} . \mathrm{s}$, in a cone-plate geometry (triangles) and in a Couette geometry with a $1 \mathrm{~mm}$ gap (circles), at $\mathrm{T}=295 \mathrm{~K}$.

of the viscosity with the shear rate (see Figure 4). At low temperature (278 K) and high $\eta_{0}$ (0.29 Pa.s), as previously described, viscosity decreases at high shear rates (Figure 4a). At intermediate temperature $(303 \mathrm{~K})$ and $\eta_{0}(0.085 \mathrm{~Pa} . \mathrm{s})$ the viscosity dependence on the shear rate is non-monotonic (Figure 4b). Finally, at high temperature $(333 \mathrm{~K})$ and low $\eta_{0}$ (0.027 Pa.s), the viscosity increases at high shear rate (Figure 4c). Such a viscosity increase for IL has already been observed in references ${ }^{19,21}$ in a cone plate geometry at high shear rate (above $1000 \mathrm{~s}^{-1}$ ), and for zero-shear viscosities below typically $50 \mathrm{mPa} . \mathrm{s}$. In those two studies, the viscosity increase is interpreted as a shear-thickening effect occurring in IL. To test this interpretation, we performed experiments in the same geometries (Couette with $1 \mathrm{~mm}$ gap and cone-plate) with a Newtonian fluid: a water-glycerol (35/65) mixture, whose nominal viscosity is close to the ones of the IL used in. ${ }^{19,21}$ As shown on Figure 4d, a viscosity 
increase is also observed with this Newtonian fluid in both geometries at shear rates above $1000 \mathrm{~s}^{-1}$.

However, Newtonian fluids such as a water-glycerol mixture are not shear-thickening. The only reasonable interpretation is that the rheometer measures a dissipation in addition to the viscous dissipation. Such an additional dissipation at high shear rates generally originates from instabilities. ${ }^{24,35}$ In the Couette geometry, this can be well explained by the Couette-Taylor instability. ${ }^{13}$ This instability generates secondary flows that increase the dissipation within the fluid. It occurs when the Taylor number $T a$ becomes higher than 1700, where $T a=\frac{\rho^{2} \dot{\gamma}^{2} h^{5}}{\eta_{0}^{2} R}$, with $\rho$ the liquid density and $R$ the radius of the inner cylinder. In our experimental conditions, $T a \simeq 2000$ for $\dot{\gamma} \simeq 2000 \mathrm{~s}^{-1}$ so that the Couette-Taylor instability can explain the viscosity increase observed in the Couette geometry (plain circle, Figure 4d).

In the cone-plate geometry, as well as in the plate-plate geometry (see the Supporting Information for more details), we interpret this apparent viscosity increase as an additional dissipation due to the fluctuations of the contact line and the radial movement of the liquid at the edges. For a typical range of shear rates from 2000 to $5000 \mathrm{~s}^{-1}$, we observe oscillations of the liquid edges anchored on surface defects (see movies in the Supporting Information). We propose that this oscillating movement originates from the competition between centrifugal forces $F_{g}$ and capillary forces $F_{\gamma}$. The centrifugal force is constant and set by the shear rate and the capillary force is assumed to vary between two extreme values $\left(F_{\gamma}^{\min }\right.$ and $\left.F_{\gamma}^{\max }\right)$ depending on the shape of the meniscus, as sketched on the scheme 1 . As $F_{g}>F_{\gamma}$, centrifugal forces expel the fluid out of the gap leading to a change of the meniscus shape and of the contact angles. This results in an increase of $F_{\gamma}$. As $F_{\gamma}$ exceeds $F_{g}$, the fluid is pulled back inside the gap. Note that such a competition between centrifugal and capillary forces has been previously reported to explain oscillations of the contact line in another geometry. ${ }^{36}$

In order to test our interpretation, we estimate the typical shear rate $\dot{\gamma}^{\star}$ for which centrifugal force $F_{g}$ and capillary force $F_{\gamma}$ are the same order of magnitude. The centrifugal 

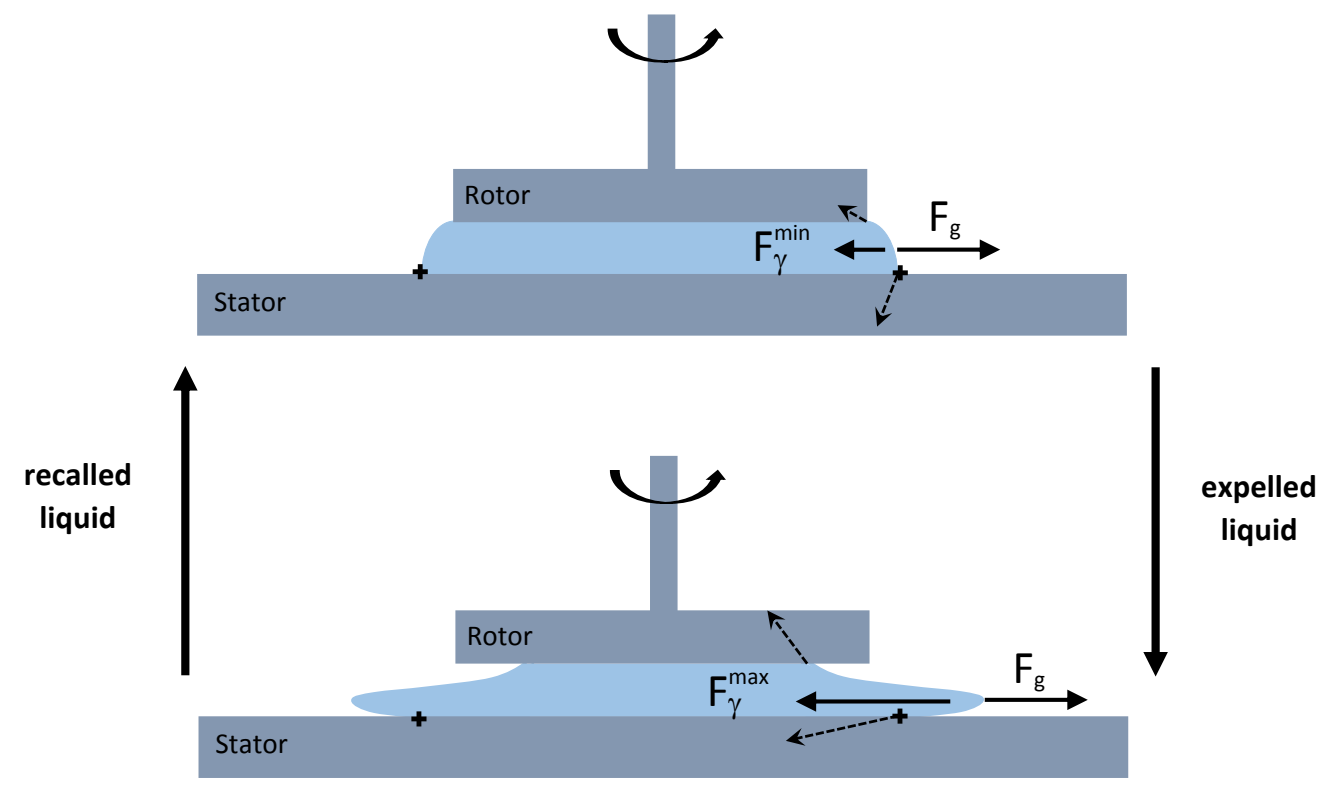

Scheme 1: Sketch of the oscillating process: competition between centrifugal and capillary forces exerted on a sheared liquid in a plate-plate geometry. The centrifugal force $F_{g}$ is constant for a given shear rate whereas the value of the capillary force $F_{\gamma}$ depends on the shape of the meniscus and oscillates between two extreme values.

force integrated on the whole cone-plate geometry is given by:

$$
F_{g}=\frac{\pi}{6} \rho R h^{3} \dot{\gamma}^{2}
$$

where $R$ and $h$ stand for the rotor radius and the gap at the extremity of the cone-plate. The capillary force exerted by the two walls on the meniscus, assuming a perfectly circular contact line at the two walls ${ }^{1}$, is given by:

$$
F_{\gamma}=4 \pi R \gamma_{L V} \cos \theta
$$

\footnotetext{
${ }^{1}$ Note that the exact value of the capillary forces depend on the exact position of the contact line and on the value of the contact angle.
} 
where $\gamma_{L V}$ and $\theta$ are respectively the surface tension of the air-liquid interface and the contact angle. Both forces are of the same order of magnitude for a shear rate:

$$
\dot{\gamma}^{\star}=\sqrt{\frac{24 \gamma_{L V} \cos \theta}{\rho h^{3}}}
$$

For classical values of surface tension $\gamma_{L V}=60 \mathrm{mN} / \mathrm{m}$ and for $\theta=0, h=1 \mathrm{~mm}, \rho=$ $10^{3} \mathrm{~kg} / \mathrm{m}^{3}$, we found $\dot{\gamma}^{\star} \simeq 1200 \mathrm{~s}^{-1}$. This is in good agreement with the typical shear rates observed experimentally as shown in Figure 4d. This analysis in terms of force balance leads to a shear rate $\dot{\gamma}^{\star}$ above which capillary instability is expected. Moreover, taking into account viscous dissipation sets a critical viscosity $\eta^{\star}$ above which this instability is damped (see the Supporting Information). Overall, this capillary instability is then observed for $\dot{\gamma}>\dot{\gamma}^{\star}$ and for $\eta<\eta^{\star}$.

Finally, instabilities and viscous heating can also coexist and compete at high shear rates, and the relative importance of these two effects depends on the shear rate and the zero shear viscosity $\eta_{0}$. Indeed, lower (resp. higher) $\eta_{0}$ promotes instabilities (resp. viscous heating). Such a competition gives an explanation for a non-monotonic dependence of viscosity on the shear rate, as observed in Figure 4b.

To summarize, we measured the increase or decrease of the viscosity at high shear rates for IL and Newtonian fluids. Those behaviors cannot be simply attributed to shear-thickening or shear-thinning effects. Indeed, rheological measurements performed at high shear rate $\dot{\gamma}>1000 \mathrm{~s}^{-1}$ have to be interpreted carefully, since many additional effects may occur. On one hand, viscous heating is at the origin of a viscosity decrease that can be misinterpreted as a shear-thinning. The resulting viscosity decrease is all the more important as the thermal sensitivity $\beta$ and the viscosity $\eta_{0}$ are high. On the other hand, instabilities (Couette-Taylor or contact line fluctuations) induce a dissipation increase, which can be misinterpreted as shear-thickening. The instability thresholds depend both on the experimental geometry and the fluid properties. Both instabilities are favored at a low viscosity $(\eta<0.1$ Pa.s in our 
experiments) and large gap. As a consequence, there is no evidence that the ILs mentioned here are non-Newtonian in the investigated range, since similar behaviors have been observed in known Newtonian liquids. A comparison with matching Newtonian liquids (similar viscosity and thermal sensitivity) should also be performed for the systems investigated in ${ }^{19-21}$ or cited in the review of Shakeel et al. ${ }^{22}$ in order to validate or invalidate the interpretation of the observed viscosity variations in terms of shear-thinning or shear-thickening. Moreover strong dependence of the rheological response on the geometry (couette, cone-plate, plate-plate) has to be considered carefully.

\section{Acknowledgement}

The authors thank Laurent Joly for fruitful discussions and Margarida Costa Gomes and Agilio Padua for introducing them to ionic liquids.

\section{Supporting Information Available}

Flow curves for $\left[\mathrm{C}_{n} \mathrm{mim}\right][\mathrm{NTF} 2]$ with $n=1,2,4,8$, Carreau fit of the normalized viscosity for $\left[\mathrm{C}_{10} \mathrm{mim}\right][\mathrm{NTF} 2]$, quantitative information about temperature increase induced by shear, supplementary information on the contact line instability, illustrated by two movies and scaling analysis of the instability damping due to viscous dissipation.

This material is available free of charge via the Internet at http://pubs.acs.org/.

\section{References}

(1) Torimoto, T.; Tsuda, T.; ichi Okazaki, K.; Kuwabata, S. New Frontiers in Materials Science Opened by Ionic Liquids. Advanced Materials 2010, 22, 1196-1221.

(2) Rogers, R. D. CHEMISTRY: Ionic Liquids-Solvents of the Future? Science 2003, 302, 792-793. 
(3) Palacio, M.; Bhushan, B. A Review of Ionic Liquids for Green Molecular Lubrication in Nanotechnology. Tribology Letters 2010, 40, 247-268.

(4) Lhermerout, R.; Diederichs, C.; Perkin, S. Are Ionic Liquids Good Boundary Lubricants? A Molecular Perspective. Lubricants 2018, 6, 9-12.

(5) Marsh, K. N.; Boxall, J. A.; Lichtenthaler, R. Room temperature ionic liquids and their mixtures - a review. Fluid Phase Equilibria 2004, 219, 93-98.

(6) Zhang, S.; Lu, X.; Zhou, Q.; Li, X.; Zhang, X.; Li, S. Ionic Liquids; Elsevier, 2009.

(7) Hayes, R.; Warr, G. G.; Atkin, R. Structure and Nanostructure in Ionic Liquids. Chemical Reviews 2015, 115, 6357-6426.

(8) Tariq, M.; Carvalho, P. J.; Coutinho, J. A.; Marrucho, I. M.; Lopes, J. N. C.; Rebelo, L. P. Viscosity of (C2-C14) 1-alkyl-3-methylimidazolium bis(trifluoromethylsulfonyl)amide ionic liquids in an extended temperature range. Fluid Phase Equilibria 2011, 301, 22-32.

(9) Fitchett, B. D.; Knepp, T. N.; Conboy, J. C. 1-Alkyl-3-methylimidazolium Bis (perfluoroalkylsulfonyl) imide water-immiscible ionic liquids the effect of water on electrochemical and physical properties. Journal of the Electrochemical Society 2004, 151, E219-E225.

(10) Voeltzel, N.; Vergne, P.; Fillot, N.; Bouscharain, N.; Joly, L. Rheology of an Ionic Liquid with Variable Carreau Exponent: A Full Picture by Molecular Simulation with Experimental Contribution. Tribology Letters 2016, 64, 25.

(11) Spikes, H. A. Comment on: Rheology of an Ionic Liquid with Variable Carreau Exponent: A Full Picture by Molecular Simulation with Experimental Contribution, by Nicolas Voeltzel, Philippe Vergne, Nicolas Fillot, Nathalie Bouscharain, Laurent Joly, Tribology Letters (2016) 64:25. Tribology Letters 2017, 65. 
(12) Safinejad, R.; Mehdipour, N.; Eslami, H. Atomistic reverse nonequilibrium molecular dynamics simulation of the viscosity of ionic liquid 1-n-butyl 3-methylimidazolium bis(trifluoromethylsulfonyl)imide [bmim][Tf2N]. Physical Chemistry Chemical Physics 2018, 20, 21544-21551.

(13) Larson, R. G. Flow-induced mixing, demixing, and phase transitions in polymeric fluids. Rheologica Acta 1992, 31, 497-520.

(14) Förster, S.; Konrad, M.; Lindner, P. Shear thinning and orientational ordering of wormlike micelles. Physical review letters 2005, 94, 017803.

(15) Seto, R.; Mari, R.; Morris, J. F.; Denn, M. M. Discontinuous shear thickening of frictional hard-sphere suspensions. Physical review letters 2013, 111, 218301.

(16) Wyart, M.; Cates, M. Discontinuous shear thickening without inertia in dense nonBrownian suspensions. Physical review letters 2014, 112, 098302.

(17) Clavaud, C.; Bérut, A.; Metzger, B.; Forterre, Y. Revealing the frictional transition in shear-thickening suspensions. Proceedings of the National Academy of Sciences 2017, $\underline{114}, 5147-5152$.

(18) Comtet, J.; Chatté, G.; Niguès, A.; Bocquet, L.; Siria, A.; Colin, A. Pairwise frictional profile between particles determines discontinuous shear thickening transition in noncolloidal suspensions. Nature communications 2017, $\underline{8}, 15633$.

(19) Burrell, G. L.; Dunlop, N. F.; Separovic, F. Non-Newtonian viscous shear thinning in ionic liquids. Soft Matter 2010, $\underline{6}, 2080-7$.

(20) Kulkarni, P.; Branco, L.; Crespo, J.; Nunes, M.; Raymundo, A.; Afonso, C. Comparison of Physicochemical Properties of New Ionic Liquids Based on Imidazolium, Quaternary Ammonium, and Guanidinium Cations. Chemistry - A European Journal 2007, 13, $8478-8488$. 
(21) Jacquemin, J.; Anouti, M.; Lemordant, D. Physico-Chemical Properties of NonNewtonian Shear Thickening Diisopropyl-ethylammonium-Based Protic Ionic Liquids and Their Mixtures with Water and Acetonitrile. Journal of Chemical \& Engineering Data 2011, 56, 556-564.

(22) Shakeel, A.; Mahmood, H.; Farooq, U.; Ullah, Z.; Yasin, S.; Iqbal, T.; Chassagne, C.; Moniruzzaman, M. Rheology of pure ionic liquids and their complex fluids: A review. ACS Sustainable Chemistry \& Engineering 2019, 7, 13586-13626.

(23) Dontula, P.; Macosko, C. W.; Scriven, L. E. Does the Viscosity of Glycerin Fall at High Shear Rates? Industrial \& Engineering Chemistry Research 1999, 38, 1729-1735.

(24) Fardin, M. A.; Perge, C.; Casanellas, L.; Hollis, T.; Taberlet, N.; Ortín, J.; Lerouge, S.; Manneville, S. Flow instabilities in large amplitude oscillatory shear: a cautionary tale.

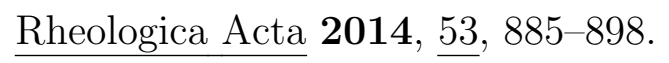

(25) Pádua, A. A.; Costa Gomes, M. F.; Canongia Lopes, J. N. Molecular solutes in ionic liquids: a structural perspective. Accounts of Chemical Research 2007, 40, 1087-1096.

(26) Shimizu, K.; Bernardes, C. E. S.; Canongia Lopes, J. N. Structure and Aggregation in the 1-Alkyl-3-Methylimidazolium Bis(trifluoromethylsulfonyl)imide Ionic Liquid Homologous Series. The Journal of Physical Chemistry B 2014, 118, 567-576, PMID: 24358956.

(27) Makino, W.; Kishikawa, R.; Mizoshiri, M.; Takeda, S.; Yao, M. Viscoelastic properties of room temperature ionic liquids. The Journal of Chemical Physics 2008, 129, 104510.

(28) Yamaguchi, T.; Nakahara, E.; Koda, S. Quantitative Analysis of Conductivity and Viscosity of Ionic Liquids in Terms of Their Relaxation Times. The Journal of Physical Chemistry B 2014, 118, 5752-5759. 
(29) Russina, O.; Triolo, A.; Gontrani, L.; Caminiti, R.; Xiao, D.; Jr, L. G. H.; Bartsch, R. A.; Quitevis, E. L.; Pleckhova, N.; Seddon, K. R. Morphology and intermolecular dynamics of 1-alkyl-3-methylimidazolium bis $\{$ (trifluoromethane)sulfonyl $\}$ amide ionic liquids: structural and dynamic evidence of nanoscale segregation. Journal of Physics: Condensed Matter 2009, 21, 424121.

(30) Nahme, R. Beitrge zur hydrodynamischen Theorie der Lagerreibung. Ing. Arch. 1940, 11, 191-209.

(31) Bird, R. B.; Turian, R. Viscous heating effects in a cone and plate viscometer. Chemical Engineering Science 1962, 17, 331-334.

(32) Pipe, C. J.; Majmudar, T. S.; McKinley, G. H. High shear rate viscometry. Rheologica Acta 2008, 47, 621-642.

(33) Turian, R.; Bird, R. B. Viscous heating effects in a cone and plate viscometer II. Chemical Engineering Science 1963, 18, 689-696.

(34) Sukanek, P. C.; Laurence, R. L. An experimental investigation of viscous heating in some simple shear flows. AIChE Journal 1974, 20, 474-484.

(35) Ewoldt, R. H.; Johnston, M. T.; Caretta, L. M. Complex fluids in biological systems; Springer, 2015; pp 207-241.

(36) Couvreur, S.; Daerr, A. The role of wetting heterogeneities in the meandering instability of a partial wetting rivulet. EPL (Europhysics Letters) 2012, 99, 24004. 